\title{
Ventromedial Prefrontal Cortex Lesions Alter Neural and Physiological Correlates of Anticipation
}

\author{
Julian C. Motzkin, ${ }^{1,2,3}$ Carissa L. Philippi, ${ }^{1}$ Richard C. Wolf, ${ }^{1,2}$ Mustafa K. Baskaya, ${ }^{4}$ and ${ }^{\circledR}$ Michael Koenigs ${ }^{1}$ \\ Departments of ${ }^{1}$ Psychiatry, ${ }^{2}$ Neuroscience Training Program, ${ }^{3}$ Medical Scientist Training Program, and ${ }^{4}$ Neurological Surgery, University of Wisconsin- \\ Madison, Madison, Wisconsin 53792
}

Uncertainty is a ubiquitous feature of our daily lives. Although previous studies have identified a number of neural and peripheral physiological changes associated with uncertainty, there are limited data on the causal mechanisms underlying these responses in humans. In this study, we address this empirical gap through a novel application of fMRI in neurosurgical patients with focal, bilateral ventromedial prefrontal cortex (vmPFC) damage. The fMRI task involved cued anticipation of aversive and neutral picture stimuli; "certain" cues unambiguously indicated the upcoming picture valence, whereas "ambiguous" cues could precede either picture type. Healthy subjects exhibited robust bilateral insula responses to ambiguous cues, and this cue-related insula activity significantly correlated with heart rate variability during the task. By contrast, the vmPFC lesion patients exhibited altered cue-related insula activity and reduced heart rate variability. These findings suggest a role for vmPFC in coordinating neural and physiological responses during anticipation.

\section{Introduction}

Acknowledging the uncertainty inherent in virtually all human endeavors, Benjamin Franklin famously quipped, "in this world nothing can be said to be certain, except death and taxes." How an individual copes with the uncertainty that pervades daily life can have a substantial impact on his or her psychological well being. Excessive worry about potential future aversive events is a core feature of anxiety disorders (APA, 1994). As recent clinical studies have highlighted the central role that intolerance of uncertainty plays in anxiety disorders (Garfinkle and Behar, 2012; McEvoy and Mahoney, 2012; Grupe and Nitschke, 2013), studies in cognitive and affective neuroscience have begun to delineate the neural circuitry involved in the anticipation of uncertain future events. Research in healthy adult populations has revealed a network of brain regions that become active during the anticipation of unpredictable or uncertain outcomes, including insula, amygdala, ventromedial/orbital prefrontal cortex, and anterior cingulate cortex (Critchley et al., 2001; Dunsmoor et al., 2007; Sarinopoulos et al., 2010; Somerville et al., 2013). These same brain areas have been widely implicated in the pathogenesis of anxiety disorders (Lorberbaum et al., 2004; Rauch et al., 2006; Simmons et al., 2006; Etkin and Wager, 2007; Nitschke et al., 2009; Grupe and Nitschke, 2013). However, the mechanisms of

Received April 9, 2014; revised June 27, 2014; accepted June 27, 2014.

Author contributions: J.C.M., M.K.B., and M.K. designed research; J.C.M. performed research; J.C.M. contributed unpublished reagents/analytic tools; J.C.M., C.L.P., and R.C.W. analyzed data; J.C.M., C.L.P., R.C.W., M.K.B., and M.K. wrote the paper.

This work was supported by Grants from the National Institutes of Health (R01MH101162, T32GM007507, T32GM008692, T32MH018931).

The authors declare no competing financial interests.

Correspondence should be addressed to Dr Michael Koenigs, University of Wisconsin-Madison, 6001 Research Park Boulevard, Madison, WI 53719. E-mail: mrkoenigs@wisc.edu.

DOI:10.1523/JNEUROSCI.1446-14.2014

Copyright $\odot 2014$ the authors $\quad 0270-6474 / 14 / 3410430-08 \$ 15.00 / 0$ interaction among these brain regions during uncertainty remain unclear.

With respect to anticipatory affective processing, the ventromedial prefrontal cortex (vmPFC) is a key node in this network. Several previous neuroimaging studies in healthy adult populations have observed vmPFC activation during the anticipation of potentially aversive stimuli, including pain, unpleasant pictures, and risky choices (Ploghaus et al., 1999; Critchley et al., 2001; Nitschke et al., 2006), with the magnitude of vmPFC activity correlating with self-reported anticipatory anxiety and negative affect (Sawamoto et al., 2000; Simpson et al., 2001). Patients with vmPFC damage reliably exhibit decision-making deficits in tasks involving uncertain or risky outcomes (Bechara et al., 1994; Fellows and Farah, 2003; Tsuchida et al., 2010), as well as diminished anticipatory arousal preceding risky, uncertain choices (Bechara et al., 1997). Anatomical connectivity data provide additional support for this view; vmPFC shares reciprocal connections with subcortical, hypothalamic, and brainstem regions responsible for somatic components of emotion, as well as with cortical regions, such as lateral prefrontal and anterior cingulate cortex, that mediate cognitive and behavioral control (Neafsey, 1990; Ongür and Price, 2000; Barbas et al., 2003; Ghashghaei et al., 2007). Together, these previous findings suggest that vmPFC may play a pivotal role in coordinating neural and peripheral physiological responses to uncertainty during the anticipation of aversive stimuli. To test this hypothesis, we used fMRI and a measure of heart rate variability (HRV) in a sample of human neurosurgical patients with focal bilateral vmPFC damage while they completed a task involving the cued anticipation of aversive and neutral picture stimuli. We predicted that vmPFC damage would significantly alter fMRI and HRV correlates of uncertainty. 


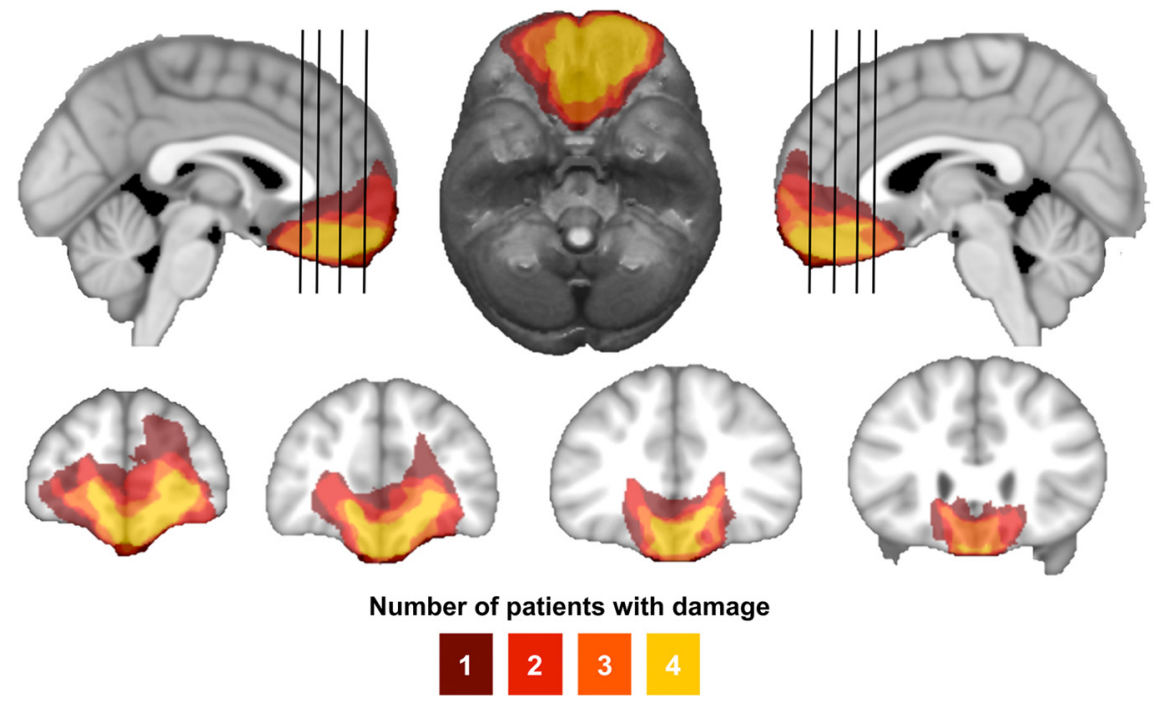

Figure 1. Lesion overlap of vmPFC patients. Color indicates the number of overlapping lesions at each voxel. All vmPFC patients had damage to the medial one-third of the orbitofrontal cortex and the ventral one-third of medial surface of prefrontal cortex, bilaterally. This area includes Brodmann areas 11, 12, 24, 25, 32, and the medial portion of 10 below the level of the genu of the corpus callosum, as well as subjacent white matter.

Table 1. Subject characteristics

\begin{tabular}{lcccccccc}
\hline & Age & Sex & Edu & IQ & Pos Aff & Neg Aff & BDI-II & STAI-T \\
\hline vmPFC & 58.5 & $3 \mathrm{M}$ & 15.5 & 103.8 & 36 & 17.0 & 7.0 & 34.3 \\
$(n=4)$ & $(6.2)$ & $1 \mathrm{~F}$ & $(4.1)$ & $(12.4)$ & $(8.4)$ & $(8.7)$ & $(3.2)$ & $(9.5)$ \\
NC & 51.7 & $11 \mathrm{M}$ & 17.7 & 110.9 & 37.8 & 13.0 & 4.0 & 31.6 \\
$(n=19)$ & $(9.9)$ & $8 \mathrm{~F}$ & $(3.5)$ & $(7.2)$ & $(4.9)$ & $(2.4)$ & $(3.3)$ & $(6.0)$ \\
$P($ vmPFC vs NC) & 0.16 & 0.63 & 0.51 & 0.25 & 0.56 & 0.73 & 0.11 & 0.44
\end{tabular}

Means are presented with SDs in parentheses. Edu, Years of education; IQ, intelligence quotient estimated by the Wide Range Achievement Test 4, Blue Reading subtest (Wilkinson and Robertson, 2006); Pos/Neg Aff, scores from the Positive and Negative Affect Schedule (Watson et al., 1988); BDI-II, Beck Depression Inventory-II (Beck et al., 1996); STAI-T, trait version of the Spielberger State Trait Anxiety Inventory (Spielberger et al., 1983).

\section{Materials and Methods}

Participants. The target lesion group consisted of four adult neurosurgical patients ( 3 males, 1 female) with extensive bilateral parenchymal damage, largely confined to the vmPFC, defined as the medial one-third of the orbital surface and the ventral one-third of the medial surface of prefrontal cortex, bilaterally (Fig. 1). Each of the four patients had undergone surgical resection of a large anterior cranial fossa meningioma via craniotomy. Initial clinical presentations included subtle or obvious personality changes over several months preceding surgery. On postsurgical MRI, although vasogenic edema largely resolved, there were persistent $\mathrm{T}_{2}$-weighted signal changes, consistent with gliosis, in the vmPFC bilaterally. All experimental procedures were conducted $>3$ months after surgery, when the expected recovery was complete. At the time of testing, all patients had focal, stable MRI signal changes and resection cavities and were free of dementia and substance abuse. Nineteen healthy adults (11 males, 8 females) with no history of brain injury, neurological or psychiatric illness, or current use of psychoactive medication were recruited as a normal comparison (NC) group. Demographic and neuropsychological data for the vmPFC and $\mathrm{NC}$ groups are summarized in Table 1.

Event-related fMRI task. During the fMRI task, which was adapted from previous studies (Sarinopoulos et al., 2010; Grupe et al., 2013), subjects viewed 64 unique images drawn from the International Affective Picture System (IAPS; Lang et al., 2008), divided evenly among pictures with aversive and neutral content. Aversive stimuli consisted of 32 negative/unpleasant and arousing images, based on published norms (Lang et al., 2008; Ewbank et al., 2009; valence: $2.01 \pm 0.39$, arousal: $6.25 \pm 0.7$ ). Neutral stimuli consisted of 32 images with neutral valence and low arousal ratings (valence: $4.96 \pm 0.21$, arousal: $2.95 \pm 0.77$ ). A list of individual stimuli is reported in a previous study (Motzkin et al., 2014).
All images were preceded by one of three visual cues ("X," "O," or "?"). The $\mathrm{X}$ and $\mathrm{O}$ cues indicated that the subsequent image would be aversive or neutral, respectively, whereas the? cue provided no information regarding the emotional content of the image (equal likelihood of aversive or neutral content). Thus, aversive $(\mathrm{X})$ and neutral $(\mathrm{O})$ cues predicted certain outcomes, whereas ambiguous (?) cues indicated uncertainty about the impending stimulus. Each experimental trial consisted of a cue presented for $2 \mathrm{~s}$, followed, after a jittered interstimulus interval (ISI; range: $2-8 \mathrm{~s}$ ), by a $1 \mathrm{~s}$ picture presentation. After a second jittered ISI (range: $5-9 \mathrm{~s}$ ), subjects had $4 \mathrm{~s}$ to rate their emotional response to the image using a 4-item scale ranging from 1 ("very positive") to 4 ("very negative"). Before scanning, subjects were informed of all cue-picture contingencies and completed a practice task consisting of 16 unique trials (4 per cue-picture pair) to ensure task comprehension.

MRI data acquisition. All structural and functional MRI data were acquired using a 3.0 T GE Discovery MR750 scanner equipped with an 8-channel radio-frequency head coil array (General Electric Medical Systems). Highresolution $\mathrm{T}_{1}$-weighted anatomical images were acquired using an inversion-recovery spoiled GRASS (SPGR) sequence $(\mathrm{TR}=8.2 \mathrm{~ms}$, TE $=$ $3.2 \mathrm{~ms}, \alpha=12^{\circ}, \mathrm{FOV}=256 \times 256 \mathrm{~mm}$, matrix $=256 \times 256$, in-plane resolution $=1 \times 1 \mathrm{~mm}^{2}$, slice thickness $=1 \mathrm{~mm}, 1024$ axial slices). To facilitate lesion segmentation, we collected a separate $\mathrm{T}_{2}$-weighted FLAIR scan $\left(\mathrm{TR}=8650 \mathrm{~ms}, \mathrm{TE}=136 \mathrm{~ms}, \alpha=0^{\circ}, \mathrm{FOV}=220 \times 220 \mathrm{~mm}^{2}\right.$, matrix $=512 \times 512$, in-plane resolution $=0.43 \times 0.43 \mathrm{~mm}^{2}$, slice thickness $=5 \mathrm{~mm}$, gap $1 \mathrm{~mm}, 25$ axial slices).

Baseline resting cerebral blood flow (CBF) was estimated using a $3 \mathrm{D}$ fast spin echo spiral sequence with pseudocontinuous arterial spin labeling (pcASL; Dai et al., 2008; Xu et al., 2010; Okonkwo et al., 2014) and background suppression for quantitative perfusion measurements $(\mathrm{TR}=4653 \mathrm{~ms}, \mathrm{TE}=10.5 \mathrm{~ms}$, postlabeling delay $=1525 \mathrm{~ms}$, labeling duration $=1450 \mathrm{~ms}, 8$ interleaved spiral arms with 512 samples at 62.5 $\mathrm{kHz}$ bandwidth and $384-\mathrm{mm}$-thick slices, number of excitations $=3$, scan duration $=4.5 \mathrm{~min}$ ).

Whole-brain functional scans were acquired using a $\mathrm{T}_{2}{ }^{*}$-weighted gradient-echo echoplanar imaging $(\mathrm{EPI})$ sequence $(\mathrm{TR}=2000 \mathrm{~ms} ; \mathrm{TE}=$ $22 \mathrm{~ms} ; \alpha=79^{\circ} ; \mathrm{FOV}=224 \times 224 \mathrm{~mm}^{2}$; matrix $=64 \times 64$, in-plane resolution $=3.5 \times 3.5 \mathrm{~mm}^{2}$, slice thickness $=3 \mathrm{~mm}$, gap $=0.5 \mathrm{~mm}, 38$ interleaved axial oblique slices). Field maps were acquired using two separate acquisitions $\left(\mathrm{TR}=600 \mathrm{~ms}, \mathrm{TE}_{1}=7 \mathrm{~ms}, \mathrm{TE}_{2}=10 \mathrm{~ms}, \alpha=60^{\circ}\right.$, $\mathrm{FOV}=240 \times 240 \mathrm{~mm}^{2}$, matrix $=256 \times 128$, slice thickness $=4 \mathrm{~mm}, 33$ axial oblique slices). Rest-state functional images were collected while subjects lay still and awake, passively viewing a fixation cross for $5 \mathrm{~min}$. The two task runs lasted $12.4 \mathrm{~min}$ each. Scans were acquired in the following order: pcASL, field map, rest, task, T1, and T2-FLAIR.

Heart rate data acquisition. Heart rate data were acquired at $100 \mathrm{~Hz}$ with General Electric's photoplethysmograph, affixed to the left index finger throughout the scan session. Heart rate data were available for $n=$ $10 \mathrm{NC}$ subjects and all $n=4 \mathrm{vmPFC}$ lesion patients during the task, and $n=11 \mathrm{NC}$ subjects and all $n=4 \mathrm{vmPFC}$ lesion patients during the resting scan.

Lesion segmentation and image normalization. Individual vmPFC lesions were visually identified and manually segmented on the $\mathrm{T}_{1}$ weighted images. Lesion boundaries were drawn to include areas with gross tissue damage or abnormal signal characteristics on $\mathrm{T}_{1}$ or $\mathrm{T}_{2}$ FLAIR images. $\mathrm{T}_{1}$-weighted images were skull-stripped, rigidly coregistered with a functional volume from each subject, then diffeomorphically aligned to the Montreal Neurological Institute (MNI) coordinate system using a Symmetric Normalization algorithm (Avants and Gee, 2004) with constrained cost-function masking to prevent warping of tissue 
within the lesion mask (Brett et al., 2001). We created the lesion overlap map (Fig. 1) by computing the sum of aligned binary lesion masks for all four vmPFC patients. Alignment parameters computed during this step were used in the subsequent normalization of all anatomical and functional data to MNI space.

fMRI task preprocessing and analysis. Data analysis was conducted using AFNI (Cox, 1996) and FSL (http://www.fmrib.ox.ac.uk/fsl) software. Individual task runs were slice time corrected, field map corrected (Jezzard and Clare, 1999), motion corrected, smoothed with a $6 \mathrm{~mm}$ fullwidth half-maximum (FWHM) Gaussian kernel, and scaled to percentage signal change. Preprocessed task data were concatenated and analyzed as previously described to separately model phasic and sustained components of anticipatory activity (Grupe et al., 2013). Phasic activity was modeled using stick regressors at the onset of each cue and sustained activity was modeled using a duration-modulated boxcar regressor, beginning at cue offset and spanning the $2-8 \mathrm{~s}$ anticipatory ISI. All six cue regressors were included in a general linear model (GLM) with additional regressors for each picture type, a single regressor for the rating period, and several regressors of no interest, including six motion covariates from rigid-body alignment (Johnstone et al., 2006) and a fourth-order polynomial to model baseline and slow signal drift. Blood oxygen level-dependent (BOLD) signal was modeled by convolving each regressor with AFNI's default canonical hemodynamic response function (HRF; gamma function). To avoid potential confounds introduced by subject motion, volumes in which $>10 \%$ of voxels were time series outliers were censored before conducting the GLM; there were no group differences in the average proportion of censored volumes $\left(\chi^{2}=2.09\right.$, $p=0.15$ ), or in mean framewise displacement (NC: $0.06 \pm 0.06 \mathrm{~mm}$, vmPFC: $0.04 \pm 0.02 \mathrm{~mm} ; W=28, p=0.44)$. Resulting whole-brain maps of voxelwise $\beta$-values for phasic responses to each cue were aligned to MNI space and resampled to $3 \mathrm{~mm}^{3}$ isotropic resolution for secondlevel analyses. BOLD responses to the aversive and neutral picture stimuli were reported in a previous study (Motzkin et al., 2014). The present study specifically examines BOLD responses to the certain or ambiguous cues preceding the picture presentation.

To identify brain regions responsive to the manipulation of certainty during the cue period, we performed a voxelwise linear mixed effects (LME) analysis on phasic cue $\beta$-values from the first-level GLMs, using only the $n=19 \mathrm{NC}$ subjects (Chen et al., 2013). Cue identity (?, X, O) was modeled as a within-subjects factor, with age (mean centered) and gender included as between-subjects covariates. General linear tests for the contrast of ambiguous (?) versus certain $(\mathrm{X}+\mathrm{O})$ cues were used to identify brain regions sensitive to uncertainty in the NC group.

To identify brain regions in which cue-related activity differed between groups, we conducted a second LME analysis including data from the four vmPFC lesion patients. This full LME model included cue (?, X, $\mathrm{O})$, group (NC vs vmPFC), and the group-by-cue interaction, as well as between-subjects covariates for age (mean centered) and gender. The group-by-cue interaction map was used to identify brain regions in which the magnitude of the phasic cue response differed between NC and vmPFC groups. All statistical maps were family wise error (FWE) corrected for multiple comparisons across the whole brain at the cluster level $\left(P_{\mathrm{FWE}}<0.05\right)$, using a height threshold of $p<0.001$ (Forman et al., 1995; Carp, 2012). A corrected $P_{\mathrm{FWE}}<0.05$ was achieved using a cluster extent threshold of 38 voxels $\left(1026 \mathrm{~mm}^{3}\right)$, calculated using Monte Carlo simulations with $3 \mathrm{dClustSim}$ in AFNI.

To visualize the time course of BOLD responses to cues within significant clusters, we conducted a second GLM, replacing the canonical HRF with a series of nine TENT functions to deconvolve the raw BOLD signal. This model yielded $\beta$-values for each of nine TRs from 0 to $16 \mathrm{~s}$ after cue onset. Because significant clusters were derived using the canonical HRF, estimated response data from the deconvolution model were used for display only.

Cerebral perfusion analysis. Quantitative CBF images from pcASL were rigidly coregistered with a $\mathrm{T}_{2}$ *-weighted EPI volume from the task scan and normalized to MNI space. Normalized CBF volumes were scaled to whole-brain CBF (after masking out the lesion in vmPFC patients) and smoothed with a $6 \mathrm{~mm}$ FWHM Gaussian kernel. To rule out differences in baseline cerebral perfusion, we examined group differences in mean whole-brain CBF, as well as differences in scaled CBF for significant clusters from the group-by-cue interaction using nonparametric MannWhitney Wilcoxon tests.

Heart rate analysis. To assess HRV, we analyzed cardiac plethysmography data to compute separate estimates of heart rate and variability for the resting and task scans. Individual cardiac $R$-spikes were identified using interactive beat detection software. Resulting interbeat interval (IBI) series were visually inspected for outliers, which were handcorrected before analysis with CMETx software (Allen et al., 2007). CMETx-derived estimates of mean heart rate and heart rate variability (logHRV: the natural-log transformed variance of the unfiltered IBI series) were estimated for each scan. Task HRV estimates for each subject were calculated by averaging estimates across both task scans.

To ascertain whether individual differences in HRV were related to neural responses to the cue stimuli, we regressed task HRV estimates on percentage signal change values extracted from clusters identified in the group-by-cue interaction LME analysis. The dependent variables were parameter estimates from the linear contrast of ambiguous vs certain cues, which we conceptualized as an index of the modulation of neural activity by cue certainty. This analysis was intended to assess whether individual differences in the modulation of neural activity by cue certainty was related to overall HRV during the task. Two separate linear regression models were conducted, one examining the strength of the relationship in the NC group alone, and a second examining the relationship across both the NC and vmPFC groups together. Group differences in both task and resting HRV were assessed using nonparametric MannWhitney Wilcoxon tests, considered significant at $p<0.05$.

\section{Results}

First, we determined whether the expectancy manipulation in our task (ambiguous versus certain cues) elicited the expected pattern of neural activity in the NC subject group. Consistent with previous studies, our whole-brain analysis revealed greater bilateral insula activity for ambiguous relative to certain cues (Fig. 2; Table 2). We also observed two clusters in bilateral lingual gyrus in which responses were greater for certain relative to ambiguous cues.

Next, we identified regions of the brain in which phasic cue responses differed between the NC and vmPFC groups (a significant group-by-cue interaction). This analysis revealed three significant clusters: bilateral insula and left dorsolateral prefrontal cortex (dlPFC; Fig. 3; Table 3). Importantly, the bilateral insula regions from the interaction analysis overlapped substantially with the uncertainty-sensitive bilateral insula clusters identified in the NC group (Fig. 4), indicating that vmPFC damage significantly altered cue-related insula activity. Follow-up analyses revealed that group-by-cue interactions were driven largely by significant group differences in response to the certain aversive cues in the left insula ( $W=0, p<0.001$ ), and by significant group differences in the right insula in response to both the certain aversive cues $(W=7, p=0.008)$ and ambiguous cues $(W=64$, $p=0.035$; Fig. 3). On average, the NC group showed greater activation to ambiguous cues, relative to certain-aversive and certain-neutral cues, in bilateral insula clusters, whereas vmPFC lesion patients exhibited the greatest difference between certainaversive and certain-neutral cues. Activity in the dlPFC cluster followed a different pattern, with consistent deactivation in response to all three cues in the NC group but cue-dependent differences in activity in the vmPFC lesion group.

To ensure that group differences in insula or dlPFC activity were not due to baseline differences in perfusion following vmPFC damage, we estimated CBF using pcASL before functional scans in all subjects. There were no significant differences between the NC and vmPFC groups in whole brain CBF $(\mathrm{NC}=$ $36.30 \pm 8.73, \mathrm{vmPFC}=40.09 \pm 13.65, W=31, p=0.611)$, nor 

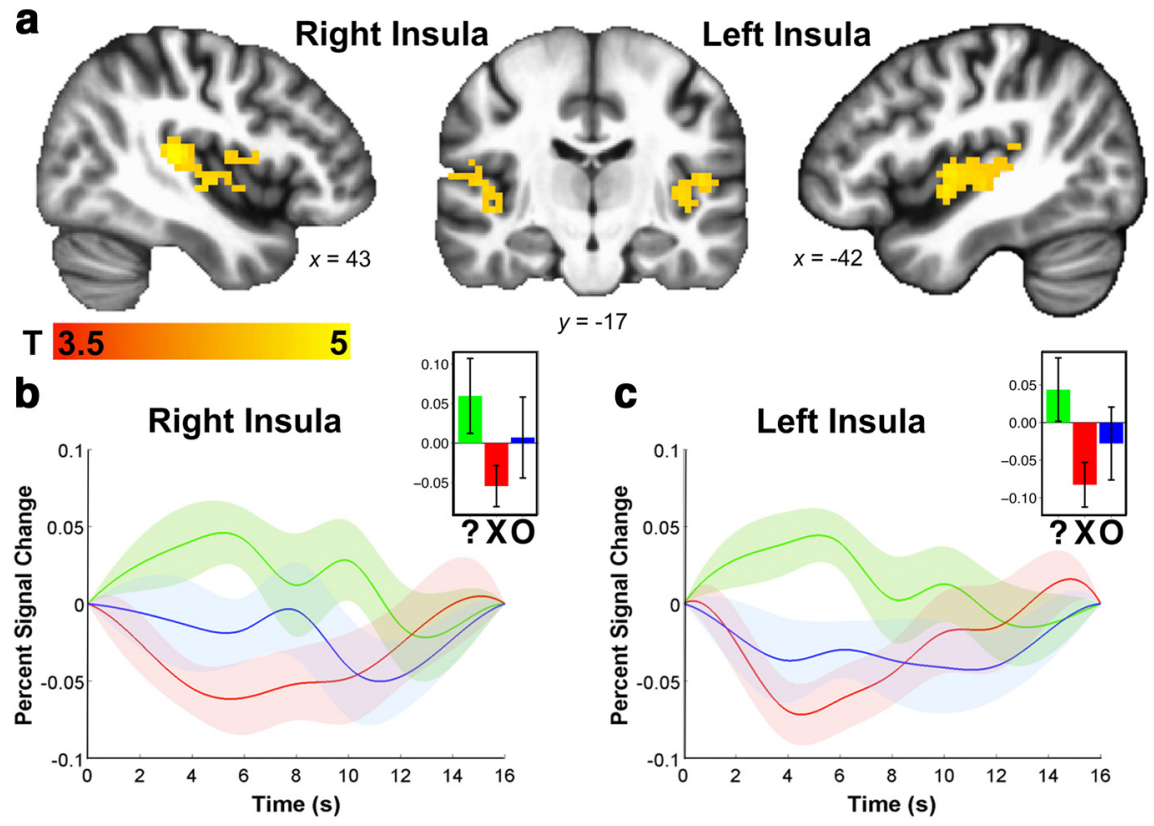

Figure 2. Brain regions with greater activation to ambiguous, relative to certain, cues in $n=19$ NC subjects. $\boldsymbol{a}$, Significant insula clusters from the ambiguous $>$ certain contrast $\left(P_{\mathrm{FWE}}<0.05\right)$. Slice coordinates (in $\mathrm{mm}$ ) are presented in MNI template space. $\boldsymbol{b}$, Mean timeseries of percentage signal change (PSC) in response in response to ambiguous (?, green), certain aversive ( $X$, red), and certain neutral ( 0 , blue) cues within the right insula cluster (width of shaded area corresponds to \pm 1 SEM). $C$, Mean time series of PSC in response to cues within the left insula cluster. Inset, The mean PSC response by cue (error bars indicate \pm 1 SEM).

Table 2. Brain regions sensitive to uncertainty in NC group

\begin{tabular}{|c|c|c|c|c|c|c|c|c|}
\hline \multirow[b]{2}{*}{ Contrast } & \multirow[b]{2}{*}{ Structure } & \multicolumn{3}{|l|}{ Cluster } & \multicolumn{4}{|c|}{ Peak voxel } \\
\hline & & $\mathrm{BA}$ & Size & $P_{\text {FWE }}$ & $T$ & $x$ & $y$ & $Z$ \\
\hline \multirow[t]{2}{*}{ Ambiguous $>$ certain } & L Insula & $13 / 41$ & 290 & $<0.001$ & 5.56 & -51 & -20 & 6 \\
\hline & RInsula & $13 / 41$ & 205 & $<0.001$ & 5.56 & 48 & -19 & 10 \\
\hline \multirow[t]{2}{*}{ Certain $>$ ambiguous } & RCuneus & 18 & 135 & $<0.001$ & -5.74 & 20 & -94 & 7 \\
\hline & L Cuneus & 18 & 48 & $<0.05$ & -4.68 & -15 & -99 & 8 \\
\hline
\end{tabular}

Cluster size in number of voxels $\left(3 \times 3 \times 3 \mathrm{~mm}^{3}\right)$. Corrected $P$ thresholds indicate minimum FWE-corrected $p$ value for each cluster. Peak voxel coordinates $(\mathrm{mm})$ are presented in MNI space. BA, Brodmann area; FWE, familywise error; $L$, left; $R$, right.

were there differences in relative $\mathrm{CBF}$ for the left insula $(\mathrm{NC}=$ $1.42 \pm 0.19, \mathrm{vmPFC}=1.35 \pm 0.15, W=45, p=0.611)$ and left dlPFC clusters $(\mathrm{NC}=0.99 \pm 0.21, \operatorname{vmPFC}=0.98 \pm 0.10$, $W=43, p=0.725$ ), although the vmPFC group had significantly lower $\mathrm{CBF}$ in the right insula cluster $(\mathrm{NC}=1.50 \pm 0.21$, $\operatorname{vmPFC}=1.29 \pm 0.12, W=63, p=0.044)$.

Finally, we investigated whether group differences in neural responses to cue stimuli were accompanied by differences in peripheral physiology. Consistent with a proposed role for vmPFC in the context-dependent modulation of peripheral physiological activity, the vmPFC lesion group had significantly lower HRV during the experimental task than the $\mathrm{NC}$ group $(\mathrm{NC}=7.23 \pm$ $0.66, \mathrm{vmPFC}=6.08 \pm 0.71, W=36, p=0.024)$. However, $\mathrm{a}$ similar group difference in $\mathrm{HRV}$ was also present during the resting $\operatorname{scan}(\mathrm{NC}=7.42 \pm 0.74, \mathrm{vmPFC}=6.14 \pm 0.55, W=41, p=$ 0.010 ), indicating a more general reduction in HRV in the vmPFC lesion group. There were no significant differences in mean heart rate between groups during either the resting or task scans (resting: $\mathrm{NC}=62.43 \pm 10.37$, vmPFC $=74.70 \pm 14.49$, $W=10, p=0.138$; task: $\mathrm{NC}=64.52 \pm 9.16$, vmPFC $=75.94 \pm$ $12.03, W=11, p=0.240$ ). To directly examine the relationship between neural responses to cues and HRV, we regressed HRV during the task on contrast estimates for ambiguous versus cer- tain cues drawn from significant clusters identified in the group-by-cue interaction analysis. For both insula clusters, larger differences between ambiguous and certain cues were associated with greater HRV during the task (both within the NC group and across the entire sample), indicating that greater sensitivity to cue certainty in bilateral insula is accompanied by greater variability in physiological reactivity during the task (Fig. 5; left insula: $r_{\mathrm{NC}-\text { only }}=0.65, p=0.043, r_{\mathrm{FULL}}=0.64$, $p=0.013$; right insula: $r_{\mathrm{NC}-\text { only }}=0.80$, $\left.p=0.005, r_{\mathrm{FULL}}=0.75, p=0.002\right)$. On average, vmPFC lesion patients exhibited patterns of neural and physiological activity consistent with the prediction line derived in the NC group, with reduced modulation of insula activity by cue certainty and lower HRV. No such relationship was present for the left dlPFC cluster, although uncertainty-related activity in this region was significantly correlated with task HRV within the NC group (left dlPFC: $r_{\mathrm{NC}-\mathrm{only}}=0.69, p=0.027, r_{\mathrm{FULL}}=$ $0.18, p=0.549)$.

\section{Discussion}

In this study, we report four main findings: (1) among NC subjects, ambiguous cues elicited stronger bilateral insula activity than certain cues, (2) vmPFC lesion patients exhibited an abnormal pattern of neural activity, particularly in bilateral insula, in response to the cues, (3) vmPFC damage was associated with attenuated HRV, and (4) across subjects, uncertainty-related insula activity was related to individual differences in HRV. Here we discuss each of these main findings in turn.

First, with respect to the finding of greater bilateral insula activity for the ambiguous versus certain cue contrast in the NC subjects, we note that this result is broadly consistent with several previous fMRI studies (Dunsmoor et al., 2007; Sarinopoulos et al., 2010; Somerville et al., 2013). Using virtually the same task, Sarinopoulos et al. (2010) found that bilateral insula responses to aversive pictures were larger following the ambiguous cue than the certain cue. Our data show a similar bilateral insula finding in response to the cues themselves. Another study using neutral and aversive pictures, which were presented in conditions of either certain/predictable temporal sequence or uncertain/unpredictable temporal sequence, found greater anterior insula activity for the uncertain condition (Somerville et al., 2013). An analogous study of Pavlovian conditioning manipulated the certainty of the pairing of the conditioned stimuli (CS) with the unconditioned stimuli (UCS) (Dunsmoor et al., 2007). Greater bilateral insula activity was observed when the CS-UCS pairing was uncertain/ unpredictable compared to when the pairing was certain/predictable. Hence, our findings bolster an emerging literature that implicates the insula in responding to the uncertain anticipation of aversive stimuli.

Next we consider the major novel finding from our study; the effect of vmPFC damage on cue-related neural activity. We observed a significant interaction between group and cue type within the same regions of mid-insular cortex that we found to be sensitive to uncertainty in the NC group, indicating that vmPFC 
lesions affect the processing of certainty in the insula. This finding supports theoretical accounts that highlight the role of vmPFC and insula in the anticipation of ambiguous, potentially aversive outcomes (Schoenbaum et al., 2009; Singer et al., 2009; Thayer et al., 2012; Grupe and Nitschke, 2013). Insula and vmPFC are reciprocally interconnected and share overlapping projections to subcortical brain regions implicated in physiological and somatic components of an emotional response (Neafsey, 1990; Allen et al., 1991; Shi and Cassell, 1998; Barbas et al., 2003). Moreover, lesions to both insula and vmPFC have been associated with abnormal patterns of decision-making under conditions of risk and uncertainty (Bechara et al., 1994; Fellows and Farah, 2003; Clark et al., 2008; Tsuchida et al., 2010).

Although the present results offer novel support for a role of vmPFC in modulating the processing of uncertainty in the insular cortex, a number of caveats regarding the fMRI data should be noted. First, the significant group-by-cue interaction in bilateral insula regions appears to be driven primarily by group differences in responses to the two certain cues; vmPFC patients exhibited abnormally elevated insula activity to the certain-aversive cues, but abnormally depressed insula activity to the certain-neutral cues. This pattern suggests that vmPFC damage yields dysregulation of anticipatory processing that is not necessarily specific to uncertainty, per se. Furthermore, the group differences in insula and dIPFC activity were especially pronounced for two of the four vmPFC lesion patients (Fig. 3). These two patients were comparable to the other two patients in terms of age and gross lesion characteristics (size, location, and chronicity), so the precise reason for this result is unclear. One limitation of the present study is the inability to determine whether vmPFC is engaged during the cue period of this task in normal subjects. Unfortunately, the area of vmPFC damage in our patient sample corresponds almost exactly to the area of maximal fMRI signal dropout due to magnetic field inhomogeneities. Hence, we are unable to determine whether the vmPFC damage disrupts local processing, or perhaps impairs communication between insula and other cortical areas via damage to underlying white matter. Finally, to account for the absence of a lesion control group, we assessed baseline cerebral perfusion. Although the ASL data indicated no gross alterations of perfusion in the vmPFC patients, there was significantly reduced perfusion in right (but not left) insula. Thus, deficient cerebral perfusion cannot readily explain the observed group-bycue interactions, which include a mix of abnormally elevated and abnormally depressed BOLD responses in the vmPFC lesion group.

Our heart rate data provide complementary evidence of the effect of vmPFC lesions on anticipatory processing. High levels of HRV are thought to subserve a greater capacity to adapt to evolving environmental demands (Thayer et al., 2012). Indeed, higher resting HRV is associated with adaptive behavioral and physiological responses and with greater emotion regulation ability
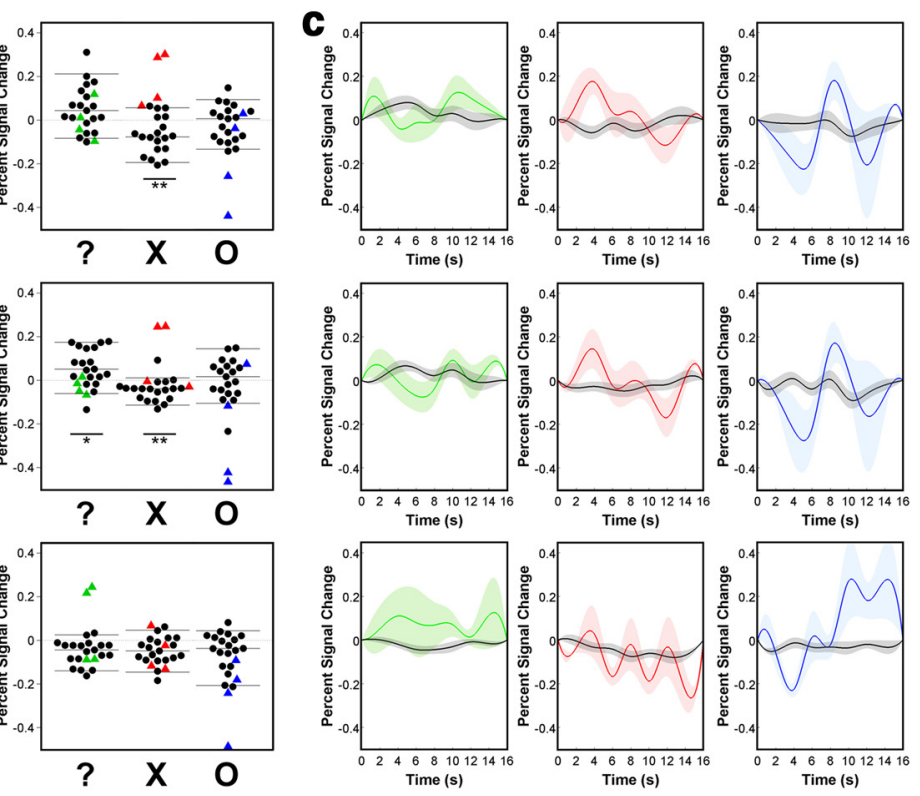

$?$

$\mathbf{0}$

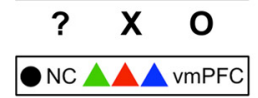

$\mathbf{x}$

(1)

Figure 3. Brain regions showing significant group-by-cue interactions $\left(P_{\mathrm{FWE}}<0.05\right)$. $\boldsymbol{a}$, Group differences in cue reactivity were observed in left insula (top row), right insula (middle row), and left dIPFC (bottom row). Slice coordinates (in mm) are presented in 列 mean time series in response to each cue type are displayed forvmPFC lesion patients (green, red, and blue ribbons) and NC subjects (gray ribbons). The width of the shaded ribbons in time series plots corresponds to \pm 1 SEM; ${ }^{*} p<0.05,{ }^{* *} p<0.01$.

Table 3. Significant clusters identified in the group-by-cue interaction analysis

\begin{tabular}{|c|c|c|c|c|c|c|c|}
\hline \multirow[b]{2}{*}{ Structure } & \multicolumn{3}{|l|}{ Cluster } & \multicolumn{4}{|c|}{ Peak voxel } \\
\hline & $B A$ & Size & $P_{\mathrm{FWE}}$ & $F$ & $x$ & $y$ & $z$ \\
\hline L Insula & 13 & 86 & $<0.001$ & 13.73 & -44 & -5 & -5 \\
\hline R Insula & $13 / 41$ & 79 & $<0.001$ & 13.26 & 46 & -8 & -2 \\
\hline L dIPFC & $8 / 9$ & 42 & $<0.05$ & 12.56 & -21 & 38 & 46 \\
\hline
\end{tabular}

Cluster size in number of voxels $\left(3 \times 3 \times 3 \mathrm{~mm}^{3}\right)$. Corrected $P$ thresholds indicate minimum FWE-corrected $p$ value for each cluster. Peak voxel coordinates $(\mathrm{mm})$ are presented in MNI space. BA, Brodmann area; FWE, familywise error; L, left; R, right.

(Appelhans and Luecken, 2006; Melzig et al., 2009; Thayer et al., 2012). Conversely, low HRV is associated with delayed recovery from psychological stressors, as well as an increased risk of depression, anxiety, and all-cause mortality (Gorman and Sloan, 2000; Weber et al., 2010). Importantly, vmPFC activity is consistently associated with heart rate changes during emotional and cognitive tasks (Lane et al., 2009; Thayer et al., 2012). Our HRV data provide unique lesion evidence for a role of vmPFC in controlling HRV; patients with vmPFC damage exhibited significantly lower HRV during both the resting scan and the experimental task. To our knowledge, this is the first study showing reduced HRV in patients with vmPFC lesions, although this finding is consistent with several previous reports demonstrating blunted physiological responses to aversive stimuli and their anticipation following vmPFC damage (Damasio et al., 1990; Bechara et al., 1997, 1999). Furthermore, the magnitude of uncertainty-related insula activation was associated with task HRV both within the NC group and across all subjects, indicating that increased sensitivity to cue certainty in the insula was related to overall HRV during the task.

Although our results suggest a role for vmPFC in modulating insula activity and peripheral physiological responses during an- 


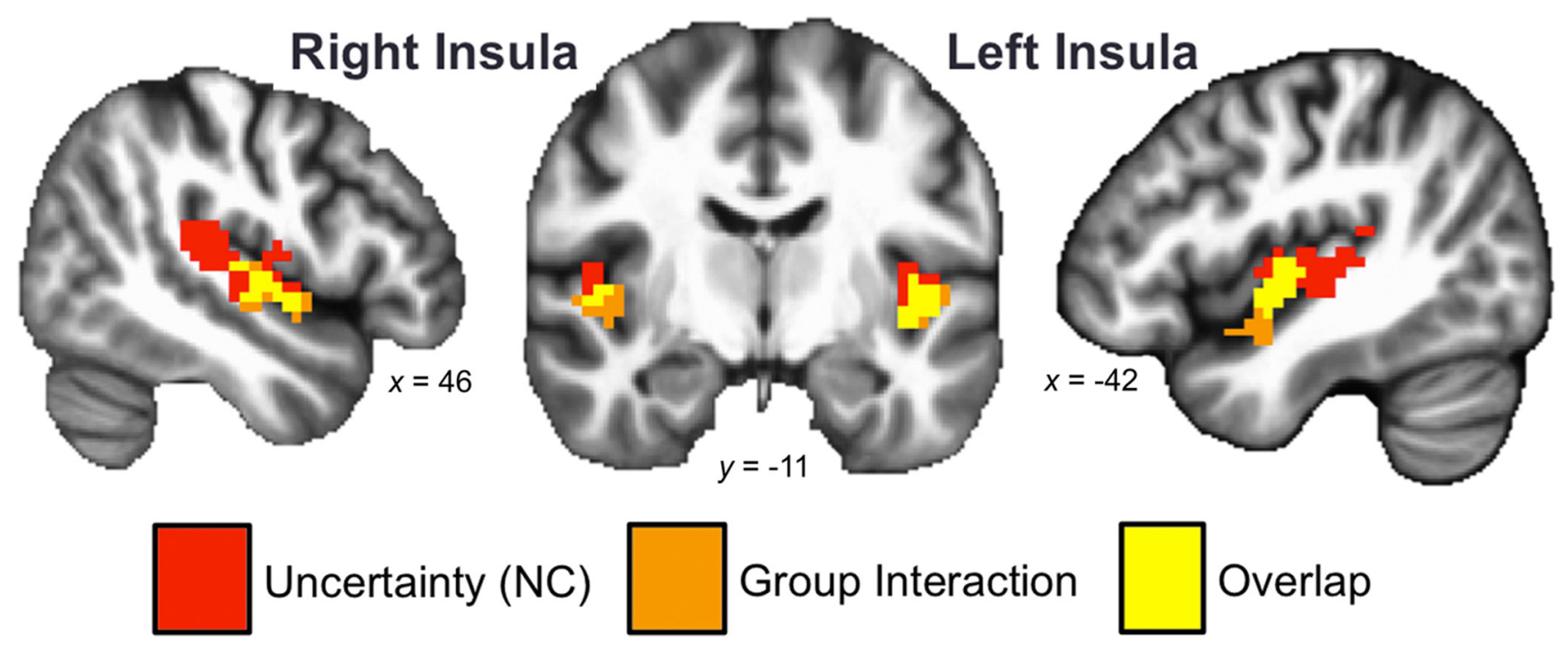

Figure 4. Conjunction analysis of regions showing significant activity in the ambiguous $>$ certain contrast in the NC group (red) and a significant group-by-cue interaction (orange). There is significant overlap in bilateral mid insula (yellow). All whole-brain maps are corrected for multiple comparisons at $P_{\text {FWE }}<0.05$. Slice coordinates (in mm) are presented in MNI template space.

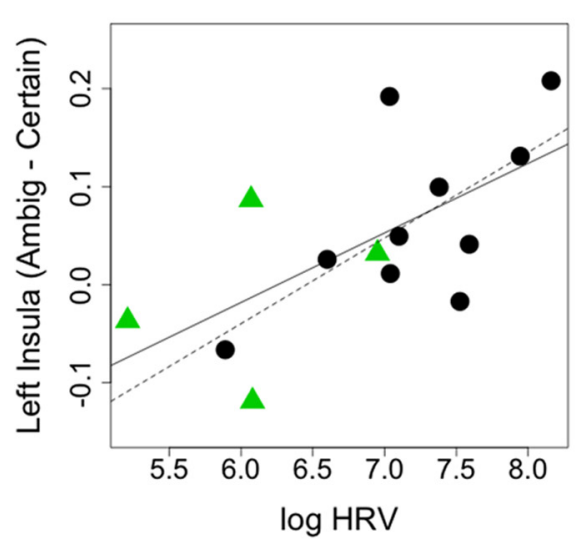

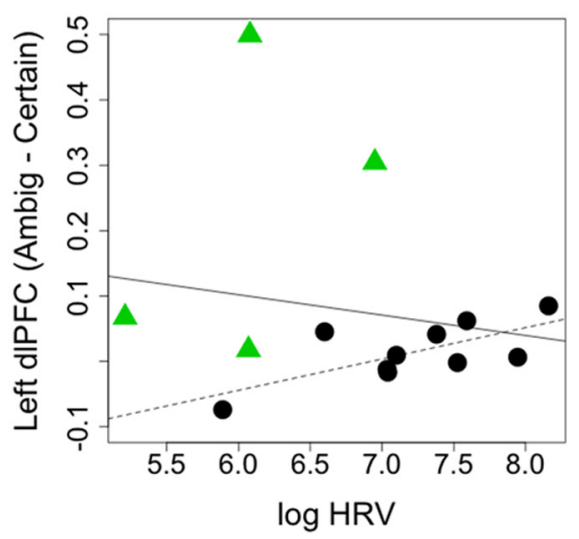

$\log \mathrm{HRV}$

NC $\triangle$ vmPFC ---- NC only — Full Group

Figure 5. Scatter plots depicting the relationship between task HRV and uncertainty-related activation (ambiguous- certain) in left insula (left), right insula (middle), and left dIPFC (right) clusters identified in the group-by-cue interaction. Green triangles represent vmPFC lesion patients and black circles represent NC subjects. Solid lines indicate the regression line across all subjects with HRV data ( $n=4 \mathrm{vmPFC}$ lesion patients, $n=10 \mathrm{NC}$ subjects), whereas dotted lines indicate the regression line for the $n=10 \mathrm{NC}$ subjects. Ambig, Ambiguous.

ticipation, the precise mechanisms underlying these effects remain unclear. One possibility is that vmPFC damage directly impairs the modulation of physiological responses, and consequently, alters somatic representations in the insular cortex. Another possibility is that vmPFC damage deprives insula of input regarding relevant contextual cues, which in turn leads to altered stimulus processing and downstream effects on physiology and behavior. Future work including patients with insular cortex lesions will be necessary to disentangle the unique contributions of each brain region to observed neural, physiological, and behavioral responses to uncertainty. Further studies could also investigate the effects of vmPFC damage on the uncertain anticipation of potentially positive outcomes, such as monetary reward, to determine whether the effects of vmPFC damage on anticipatory processing reported here are specifically related to potentially aversive stimuli, or whether they relate to uncertain anticipation more generally. In addition, future studies could include a behavioral component in the cue period to assess affective responses to the cues themselves.
In addition to the hypothesized effects in insula, our analysis also revealed a significant group-by-cue interaction in left dlPFC. In this region, vmPFC lesion patients exhibited heightened responses to ambiguous cues, but depressed responses to certainneutral cues. Interestingly, this pattern of abnormal dlPFC responses did not match the pattern of abnormal insula responses (Fig. 3), nor did the left dlPFC activity in the vmPFC patients follow the normal linear relationship with HRV, as was observed for bilateral insula activity (Fig. 5). These results suggest that dIPFC is likely not performing some simple compensatory function following vmPFC damage; instead, dlPFC may interact with vmPFC and insula during anticipatory processing. Lateral prefrontal cortex has previously been shown to be active during the anticipation of aversive stimuli (Nitschke et al., 2006; Aupperle et al., 2012), and given the dense reciprocal connections among dlPFC, vmPFC, and insula (Yeterian et al., 2012), and the putative involvement of dlPFC in goal maintenance, response selection, and attentional allocation (Miller and Cohen, 2001), dlPFC may be an important node of this circuit. 
One feature of this study that warrants consideration is the limited sample size of vmPFC lesion patients $(n=4)$. For this study, we used extremely stringent selection criteria for our target group; lesions had to involve substantial portions of vmPFC bilaterally, but could not extend significantly outside vmPFC. Furthermore, because the study involved fMRI, we could not include patients with metallic implants, such as aneurysm clips. To meet these criteria, we selected a group of patients who had all undergone surgical resection of large orbital meningiomas. So, although our sample size may be small by conventional vmPFC lesion patient standards (which typically feature $n=5$ to $n=12$ vmPFC lesion patients), it is unique with respect to the homogeneity of etiology, uniformity and selectivity of bilateral vmPFC lesions, and compatibility with fMRI.

In sum, these findings suggest a role for vmPFC in coordinating neural and physiological responses during anticipation. Our results offer new insight into the functional interactions between vmPFC and insula, two key components of the brain circuitry underlying human affective function.

\section{References}

Allen GV, Saper CB, Hurley KM, Cechetto DF (1991) Organization of visceral and limbic connections in the insular cortex of the rat. J Comp Neurol 311:1-16. CrossRef Medline

Allen JJ, Chambers AS, Towers DN (2007) The many metrics of cardiac chronotropy: a pragmatic primer and a brief comparison of metrics. Biol Psychol 74:243-262. CrossRef Medline

APA (1994) Diagnostic and statistical manual of mental disorders 4th ed. Washington DC: American Psychiatric.

Appelhans BM, Luecken LJ (2006) Heart rate variability as an index of regulated emotional responding. Rev Gen Psychol 10:229. CrossRef

Aupperle RL, Allard CB, Grimes EM, Simmons AN, Flagan T, Behrooznia M, Cissell SH, Twamley EW, Thorp SR, Norman SB, Paulus MP, Stein MB (2012) Dorsolateral prefrontal cortex activation during emotional anticipation and neuropsychological performance in posttraumatic stress disorder. Arch Gen Psychiatry 69:360-371. CrossRef Medline

Avants B, Gee JC (2004) Geodesic estimation for large deformation anatomical shape averaging and interpolation. Neuroimage 23:S139-S150. CrossRef Medline

Barbas H, Saha S, Rempel-Clower N, Ghashghaei T (2003) Serial pathways from primate prefrontal cortex to autonomic areas may influence emotional expression. BMC Neurosci 4:25. CrossRef Medline

Bechara A, Damasio AR, Damasio H, Anderson SW (1994) Insensitivity to future consequences following damage to human prefrontal cortex. Cognition 50:7-15. CrossRef Medline

Bechara A, Damasio H, Tranel D, Damasio AR (1997) Deciding advantageously before knowing the advantageous strategy. Science 275:12931295. CrossRef Medline

Bechara A, Damasio H, Damasio AR, Lee GP (1999) Different contributions of the human amygdala and ventromedial prefrontal cortex to decisionmaking. J Neurosci 19:5473-5481. Medline

Beck AT, Steer RA, Brown GK (1996) Manual for the Beck Depression Inventory II. San Antonio, TX: Psychological Corporation.

Brett M, Leff AP, Rorden C, Ashburner J (2001) Spatial normalization of brain images with focal lesions using cost function masking. Neuroimage 14:486-500. CrossRef Medline

Carp J (2012) The secret lives of experiments: methods reporting in the fMRI literature. Neuroimage 63:289-300. CrossRef Medline

Chen G, Saad ZS, Britton JC, Pine DS, Cox RW (2013) Linear mixed-effects modeling approach to FMRI group analysis. Neuroimage 73:176-190. CrossRef Medline

Clark L, Bechara A, Damasio H, Aitken MR, Sahakian BJ, Robbins TW (2008) Differential effects of insular and ventromedial prefrontal cortex lesions on risky decision-making. Brain 131:1311-1322. CrossRef Medline

Cox RW (1996) AFNI: software for analysis and visualization of functional magnetic resonance neuroimages. Comput Biomed Res 29:162-173. CrossRef Medline

Critchley HD, Mathias CJ, Dolan RJ (2001) Neural activity in the human brain relating to uncertainty and arousal during anticipation. Neuron 29:537-545. CrossRef Medline

Dai W, Garcia D, de Bazelaire C, Alsop DC (2008) Continuous flow-driven inversion for arterial spin labeling using pulsed radio frequency and gradient fields. Magn Reson Med 60:1488-1497. CrossRef Medline

Damasio AR, Tranel D, Damasio H (1990) Individuals with sociopathic behavior caused by frontal damage fail to respond autonomically to social stimuli. Behav Brain Res 41:81-94. CrossRef Medline

Dunsmoor JE, Bandettini PA, Knight DC (2007) Impact of continuous versus intermittent CS-UCS pairing on human brain activation during Pavlovian fear conditioning. Behav Neurosci 121:635-642. CrossRef Medline

Etkin A, Wager TD (2007) Functional neuroimaging of anxiety: a metaanalysis of emotional processing in PTSD, social anxiety disorder, and specific phobia. Am J Psychiatry 164:1476-1488. CrossRef Medline

Ewbank MP, Barnard PJ, Croucher CJ, Ramponi C, Calder AJ (2009) The amygdala response to images with impact. Soc Cogn Affect Neurosci 4:127-133. CrossRef Medline

Fellows LK, Farah MJ (2003) Ventromedial frontal cortex mediates affective shifting in humans: evidence from a reversal learning paradigm. Brain 126:1830-1837. CrossRef Medline

Forman SD, Cohen JD, Fitzgerald M, Eddy WF, Mintun MA, Noll DC (1995) Improved assessment of significant activation in functional magnetic resonance imaging (fMRI): use of a cluster-size threshold. Magn Reson Med 33:636-647. CrossRef Medline

Garfinkle EJ, Behar E (2012) Advances in psychotherapy for generalized anxiety disorder. Curr Psychiatry Rep 14:203-210. CrossRef Medline

Ghashghaei HT, Hilgetag CC, Barbas H (2007) Sequence of information processing for emotions based on the anatomic dialogue between prefrontal cortex and amygdala. Neuroimage 34:905-923. CrossRef Medline

Gorman JM, Sloan RP (2000) Heart rate variability in depressive and anxiety disorders. Am Heart J 140:S77-S83. CrossRef Medline

Grupe DW, Nitschke JB (2013) Uncertainty and anticipation in anxiety: an integrated neurobiological and psychological perspective. Nat Rev Neurosci 14:488-501. CrossRef Medline

Grupe DW, Oathes DJ, Nitschke JB (2013) Dissecting the anticipation of aversion reveals dissociable neural networks. Cereb Cortex 23:18741883. CrossRef Medline

Jezzard P, Clare S (1999) Sources of distortion in functional MRI data. Hum Brain Mapp 8:80-85. CrossRef Medline

Johnstone T, Ores Walsh KS, Greischar LL, Alexander AL, Fox AS, Davidson RJ, Oakes TR (2006) Motion correction and the use of motion covariates in multiple-subject fMRI analysis. Hum Brain Mapp 27:779-788. CrossRef Medline

Lane RD, McRae K, Reiman EM, Chen K, Ahern GL, Thayer JF (2009) Neural correlates of heart rate variability during emotion. Neuroimage 44 213-222. CrossRef Medline

Lang PJ, Bradley MM, Cuthbert BN (2008) International affective picture system (IAPS): affective ratings of pictures and instruction manual. Technical Report A-8. Gainesville, FL: University of Florida.

Lorberbaum JP, Kose S, Johnson MR, Arana GW, Sullivan LK, Hamner MB, Ballenger JC, Lydiard RB, Brodrick PS, Bohning DE, George MS (2004) Neural correlates of speech anticipatory anxiety in generalized social phobia. Neuroreport 15:2701-2705. Medline

McEvoy PM, Mahoney AE (2012) To be sure, to be sure: intolerance of uncertainty mediates symptoms of various anxiety disorders and depression. Behav Ther 43:533-545. CrossRef Medline

Melzig CA, Weike AI, Hamm AO, Thayer JF (2009) Individual differences in fear-potentiated startle as a function of resting heart rate variability: implications for panic disorder. Int J Psychophysiol 71:109-117. CrossRef Medline

Miller EK, Cohen JD (2001) An integrative theory of prefrontal cortex function. Annu Rev Neurosci 24:167-202. CrossRef Medline

Motzkin J, Philippi CL, Wolf RC, Baskaya MK, Koenigs M (2014) Ventromedial prefrontal cortex is critical for the regulation of amygdala activity in humans. Biol Psychiatry, in press. CrossRef

Neafsey EJ (1990) Prefrontal cortical control of the autonomic nervous system: anatomical and physiological observations. Prog Brain Res 85: 147-165; discussion 165-166. Medline

Nitschke JB, Sarinopoulos I, Mackiewicz KL, Schaefer HS, Davidson RJ (2006) Functional neuroanatomy of aversion and its anticipation. Neuroimage 29:106-116. CrossRef Medline

Nitschke JB, Sarinopoulos I, Oathes DJ, Johnstone T, Whalen PJ, Davidson 
RJ, Kalin NH (2009) Anticipatory activation in the amygdala and anterior cingulate in generalized anxiety disorder and prediction of treatment response. Am J Psychiatry 166:302-310. CrossRef Medline

Okonkwo OC, Xu G, Oh JM, Dowling NM, Carlsson CM, Gallagher CL, Birdsill AC, Palotti M, Wharton W, Hermann BP, LaRue A, Bendlin BB, Rowley HA, Asthana S, Sager MA, Johnson SC (2014) Cerebral blood flow is diminished in asymptomatic middle-aged adults with maternal history of Alzheimer's disease. Cereb Cortex 24:978-988. CrossRef Medline

Ongür D, Price JL (2000) The organization of networks within the orbital and medial prefrontal cortex of rats, monkeys and humans. Cereb Cortex 10:206-219. CrossRef Medline

Ploghaus A, Tracey I, Gati JS, Clare S, Menon RS, Matthews PM, Rawlins JN (1999) Dissociating pain from its anticipation in the human brain. Science 284:1979-1981. CrossRef Medline

Rauch SL, Shin LM, Phelps EA (2006) Neurocircuitry models of posttraumatic stress disorder and extinction: human neuroimaging researchpast, present, and future. Biol Psychiatry 60:376-382. CrossRef Medline

Sarinopoulos I, Grupe DW, Mackiewicz KL, Herrington JD, Lor M, Steege EE, Nitschke JB (2010) Uncertainty during anticipation modulates neural responses to aversion in human insula and amygdala. Cereb Cortex 20:929-940. CrossRef Medline

Sawamoto N, Honda M, Okada T, Hanakawa T, Kanda M, Fukuyama H, Konishi J, Shibasaki H (2000) Expectation of pain enhances responses to nonpainful somatosensory stimulation in the anterior cingulate cortex and parietal operculum/posterior insula: an event-related functional magnetic resonance imaging study. J Neurosci 20:7438-7445. Medline

Schoenbaum G, Roesch MR, Stalnaker TA, Takahashi YK (2009) A new perspective on the role of the orbitofrontal cortex in adaptive behaviour. Nat Rev Neurosci 10:885-892. CrossRef Medline

Shi CJ, Cassell MD (1998) Cortical, thalamic, and amygdaloid connections of the anterior and posterior insular cortices. J Comp Neurol 399:440468. CrossRef Medline

Simmons A, Strigo I, Matthews SC, Paulus MP, Stein MB (2006) Anticipation of aversive visual stimuli is associated with increased insula activation in anxiety-prone subjects. Biol Psychiatry 60:402-409. CrossRef Medline Simpson JR Jr, Drevets WC, Snyder AZ, Gusnard DA, Raichle ME (2001)
Emotion-induced changes in human medial prefrontal cortex: II. During anticipatory anxiety. Proc Natl Acad Sci U S A 98:688-693. CrossRef Medline

Singer T, Critchley HD, Preuschoff K (2009) A common role of insula in feelings, empathy and uncertainty. Trends Cogn Sci 13:334-340. CrossRef Medline

Somerville LH, Wagner DD, Wig GS, Moran JM, Whalen PJ, Kelley WM (2013) Interactions between transient and sustained neural signals support the generation and regulation of anxious emotion. Cereb Cortex 23:49-60. CrossRef Medline

Spielberger CD, Gorsuch RL, Lushene R, Vagg PR, Jacobs GA (1983) Manual for the state-trait anxiety inventory. Palo Alto, CA: Consulting Psychologists.

Thayer JF, Ahs F, Fredrikson M, Sollers JJ 3rd, Wager TD (2012) A metaanalysis of heart rate variability and neuroimaging studies: implications for heart rate variability as a marker of stress and health. Neurosci Biobehav Rev 36:747-756. CrossRef Medline

Tsuchida A, Doll BB, Fellows LK (2010) Beyond reversal: a critical role for human orbitofrontal cortex in flexible learning from probabilistic feedback. J Neurosci 30:16868-16875. CrossRef Medline

Watson D, Clark LA, Tellegen A (1988) Development and validation of brief measures of positive and negative affect: the PANAS scales. J Pers Soc Psychol 54:1063-1070. CrossRef Medline

Weber CS, Thayer JF, Rudat M, Wirtz PH, Zimmermann-Viehoff F, Thomas A, Perschel FH, Arck PC, Deter HC (2010) Low vagal tone is associated with impaired post stress recovery of cardiovascular, endocrine, and immune markers. Eur J Appl Physiol 109:201-211. CrossRef Medline

Wilkinson GS, Robertson GJ (2006) WRAT4: wide range achievement test. Lutz, FL: Psychological Assessment Resources.

Xu G, Rowley HA, Wu G, Alsop DC, Shankaranarayanan A, Dowling M, Christian BT, Oakes TR, Johnson SC (2010) Reliability and precision of pseudo-continuous arterial spin labeling perfusion MRI on 3.0 T and comparison with 15O-water PET in elderly subjects at risk for Alzheimer's disease. NMR Biomed 23:286-293. CrossRef Medline

Yeterian EH, Pandya DN, Tomaiuolo F, Petrides M (2012) The cortical connectivity of the prefrontal cortex in the monkey brain. Cortex 48:58-81. CrossRef Medline 